\title{
Detection and Phylogenetic Analysis of Human Papilloma Virus in Urine from a Sample of Iraqi Women with Vaginal Discharge
}

\author{
Ali D. Marhash¹, Qasim S. Al-Mayah², and Enas A.A. Al-Kazaaly ${ }^{3}$ \\ ${ }^{1}$ Al-Furat Al-Awsat Technical University-Babylon Institute. ${ }^{2}$ Medical Research Unit-College of Medicine, Al- \\ Nahrain University. ${ }^{3}$ Department of Obstetrics and Gynecology, College of Medicine. Al-Nahrain University, \\ Baghdad, Iraq.
}

\begin{abstract}
Human papillomavirus (HPV) is the principle cause of cervical carcinoma (CC). Detection and screening programs for this malignancy is formerly based on cytological examination of cervical cells. However, the recent trend is for detection of HPV DNA in cervical swabs (CSs). In either cases, the collection of specimens require pelvic examination which is unfavorable for women and time consuming for healthcare providers. Using urine sample for HPV DNA detection is a very good alternative; however, the efficiency of such samples in this regard is questionable. This cross-sectional study aimed to evaluated the efficiency of urine samples in detection of HPV DNA compared with CSs, and to determine the genetic relatedness of local HPV isolates from urine and CSs through phylogenetic analysis. Pair samples of first voided urine and CSs were collected from a total of $\mathbf{1 0 0}$ married women with child bearing age suffering from vaginal discharge. Cervical swabs were kept at $-20^{\circ} \mathrm{C}$ until be used, while urine samples were processed at the same day of collection. From both specimens, viral DNA was extracted and two sets of universal primers were used to amplify L1 capsid gene in a nested-PCR, the products of which were subjected for direct sequencing. The resultant sequences were aligned with reference sequences in Gen Bank. MEGA 6 software was used to construct the phylogenetic tree from local isolates and 19 reference sequences. HPV DNA was detected in $13 \%$ and $10 \%$ of CSs and urine samples respectively. Phylogenetic analysis for local HPV isolates from cervical samples revealed that 8 (61.54\%) isolates were low risk (LR) HPV. The other five isolates were high risk (HR) HPV. Kappa statistic revealed 0.853 concordance between urine samples and CSs in HPV DNA detection (95\% confidence interval $(\mathrm{Cl})=0.0 .696-1.01, \mathrm{P}<0.001)$, with $96.7 \%$ sensitivity and $100 \%$ specificity. These data strongly indicated the efficiency of urine sample in HPV detection with excellent sensitivity and specificity. Thus, urine samples could be used for routine detection and screening for HPV infection in women with child-bearing age.
\end{abstract}

Keywords: Human Papilloma Virus, Urine samples, malignancy, Iraqi women, vaginal discharge.

*Correspondence: sciencefond2015@gmail.com

(Received: 15 September 2018; accepted: 09 November 2018)

Citation: Ali D. Marhash, Qasim S. Al-Mayah, Enas A.A. Al-Kazaaly, Detection and Phylogenetic Analysis of Human Papilloma Virus in Urine from a Sample of Iraqi Women with Vaginal Discharge, J Pure Appl Microbiol., 2018; 12(4):2183-2192. http://dx.doi. org/10.22207/JPAM.12.4.57

(c) The Author(s) 2018. Open Access. This article is distributed under the terms of the Creative Commons Attribution 4.0 International License which permits unrestricted use, sharing, distribution, and reproduction in any medium, provided you give appropriate credit to the original author(s) and the source, provide a link to the Creative Commons license, and indicate if changes were made. 


\section{INTRODUCTION}

Human papillomavirus is a group of more than 200 related viruses which can be distinguished by their genetic variability ${ }^{1}$. Globally, this virus is one of the most common cause of sexually transmitted diseases (STDs) in both men and women ${ }^{2}$. According to their association with cancers, HPV types were categorized in to low risk (LR) HPV and high risk (HR) HPV. High risk HPV is thought to be the principle cause of human cervical cancer ${ }^{4}$, a considerable proportion of other anogenital cancers ${ }^{5}$, and oropharyngeal cancers $^{6}$.

Cervical cancer (CC) is the fourth most common cancer worldwide, with an estimated 528000 new cases and 26600 deaths $^{9}$. Of note, the majority of cases are squamous cell carcinoma and adenocarcinoma. About $85 \%$ of these cases occur in low and middle-income developing countries ${ }^{10}$. In Iraq, cervical cancer is less common than other developing countries and ranked tenth among women age 15 to 45 -year-old, with about 300 annual cases ${ }^{11}$.

Studies showed that early detection HPV in urogenital specimens through screening programs could significantly decrease the occurrence of CC. It was estimated that a woman needs only several screens in her lifetime to achieve this task ${ }^{12}$. A remarkable decline both in the CC-related morbidity and mortality was reported during the last decades in many high-income countries as a result of screening programs ${ }^{4}$. World Health Organization (WHO) has recommended such programs in a (screen and treat) manner strategy for women with child-bearing age ${ }^{13}$.

Screening programs were initially based on Papanicolaou (Pap) and direct molecular detection of HPV from different urogenital specimens ${ }^{14}$. In either cases, there will be a need for pelvic examination. Such procedure has many disadvantages especially in conservative society like Iraqi. It is invasive and uncomfortable for the women and cannot be carried out in settings with poor resources. Moreover, it is time consuming for healthcare provider ${ }^{15}$. Therefore, it is not feasible for screening test, at least, among Iraqi population. A proper alternative is using urine sample for HPV DNA detection. Using this alternative has many advantages, among which are urine sampling is preferred and acceptable by all women, it is non-invasive and can be self-collected, there is no need for pelvic examination, and finally many other sexually transmitted infections can be detected simultaneously ${ }^{16}$. However, there are many drawbacks in this procedure: urine is diluted and contain polymerase chain reaction (PCR) inhibitors like urea and nitrites and HPV DNA can be deteriorated by contaminating bacteria and/or endonuclease ${ }^{17}$.

\section{MATERIALS AND METHODS}

A cross-sectional study including 100 child-bearing age women suffering from vaginal discharge was conducted. Those women were attending the department of Gynecology at AlImamain Al- Kadhumain Medical City/Baghdad from December 2017 to April 2018. Inclusion criteria include married women within childbearing age suffering from vaginal discharge. Exclusion criteria include treated cases of cervical cancer, pregnant women and women with hysterectomy. Data were taken from each women including age, age at sexual initiation, total life time sex partner, number of wives for a husband,

Table 1. Total number of women and demographic distribution.

\begin{tabular}{ll}
\hline Variable & No (\%) \\
\hline Age (years) & \\
$20-30$ & $28(28 \%)$ \\
$31-40$ & $38(38 \%)$ \\
$41-50$ & $34(34 \%)$ \\
Age at marriage (years) & $12(12 \%)$ \\
$\geq 25$ & $88(88 \%)$ \\
$<25$ & \\
Duration of sexual activity & $75(75 \%)$ \\
$\geq 10$ years & $25(25 \%)$ \\
$<10$ years & $69(69 \%)$ \\
Number of wives of husband & $31(31 \%)$ \\
One & \\
Two & $8(8 \%)$ \\
Previous marriage & $92(92 \%)$ \\
Yes & \\
No & $94(94 \%)$ \\
Genital warts & $6(6 \%)$ \\
Absent & \\
Present & $65(65 \%)$ \\
Dwelling & $35(35 \%)$ \\
Rural & \\
Urbans & www.microbiologyjournal.org \\
\hline$\quad$ &
\end{tabular}


a previous marriage, and cervical examination for presence of warts in genital tract.

The present study was approved by the ethical committee of the College of Medicine/AlNahrain University.

\section{Samples collection Urine}

From each woman, $20-30 \mathrm{ml}$ of first voided urine were collected in a sterile container, transported to the lab, and centrifuged at 14000 rpm for 20 minutes to give urine sediment. DNA was extracted immediately from sediments with a ready extraction kit.

\section{Cervical Swabs}

Special endocervical swab was taken from the patient by a gynecologist and put in ice back or stored in $-20^{\circ} \mathrm{C}$ until be used.

Extraction of genomic DNA from urine and CS was done using a commercial ready kit (viral DNA extraction $\alpha$ (Genaid/Taiwan) according to the manufacturer's instructions.

Two sets of primers MY09/MY11 and GP5+/GP6+ primers, that amplify the L1 region of viral genome were used in a nested PCR. MY09/ MY11 primers amplify approximately $450 \mathrm{bp}$ of the gene, while the pair of GP5+/GP6+ amplify 150 $\mathrm{bp}$, which is internal to the sequence flanked by the former primers.

The second polymerase chain reaction products of L1 gene were directly sequenced (Macrogen/Korea). The obtained sequences were aligned with normal sequence from GenBank.

\section{Data Analysis}

Statistical package for social science (SPSS) was used for all statistical analyses. Variables were expressed as frequency and percentage and analyzed with Chi-square Phylogenic relevance between local isolates and reference isolates and within local isolates were calculated using MEGA 6 software with 1000 bootstrap replicates. A p-value of $<0.05$ was considered statistically significant.

\section{RESULTS}

\section{Demographic Data of the study population}

The present study enrolled 100 women suffering for vaginal discharge. Twelve percent from women were more than 25 years at marriage. Seventy five percent have sexual activity more than 10 years. About one third of women in current study have second-wife. Only $8 \%$ of women have a previous marriage. Warts represent approximately
$6 \%$ of study cases. About two-third (65\%) of studied women were from rural regions (Table 1 ). Molecular Detection

Gel electrophoresis of PCR product for $\mathrm{L} 1$ gene was shown in figures (1) and (2) respectively.

\section{Sequence Alignment}

PCR products from the second round for both CSs and urine samples were subjected to direct sequencing. The resultant sequences were undergone BLAST in NCBI web page, according which 19 somewhat similar sequences were chosen for the alignment and for construction of phylogenetic tree with the isolates belonging to the current study. The accession numbers of reference sequences were AF548837.1, DQ448211.1 (type 18), AF548824.1, DQ448197.1, LC155223.1 (type 16), HG793863.1, KM01523.1 (type 6), FN70704.1

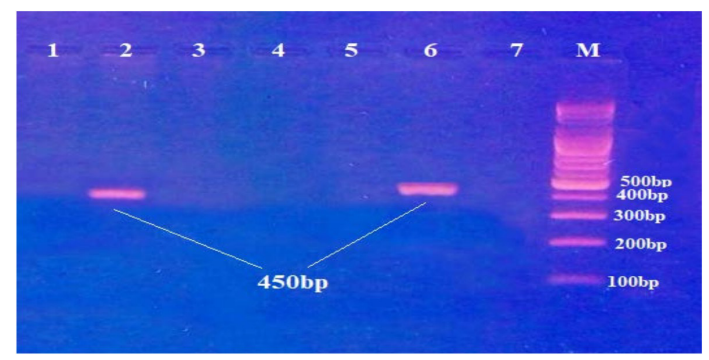

Fig. 1. Gel electrophoresis of nested- PCR products of Human Papillomavirus L1 capsid gene (the first round) stained with ethidium bromide and visualized under ultra-violate. M: DNA marker, Lanes 2 and 6: positive results for amplification (450bp). Lanes 1,3,4,5 and 7: negative results.

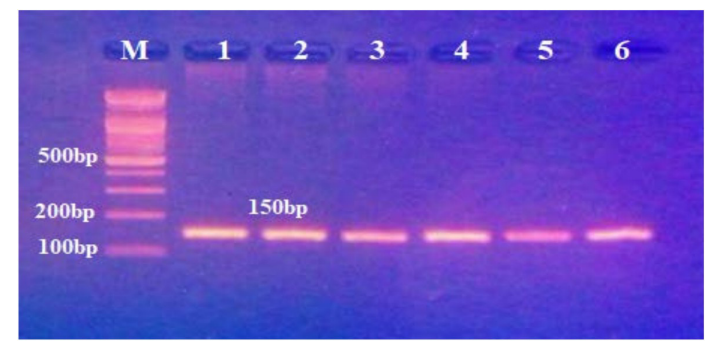

Fig. 2. Gel electrophoresis of nested-PCR products of Human Papillomavirus L1 capsid gene (the second round) stained with ethidium bromide and visualized under ultra-violate. M: DNA marker, Lanes 1,2,3,4,5 and 6: positive results for amplification (150bp). Out of 100 CSs, 13 (13\%) were found to be positive for HPV DNA. On the other hand, HPV DNA was successfully isolated from 10 samples $(10 \%)$ out of 100 urine samples collected from the same women. 
AF548824.1 type 16 Q448197.1 type 16 DQ448211.1 type 18 AF548837.1 type 18 HG793863.1 type 6 KM501523.1 type 6 FN870704.1 type 11 KC470250.1 type 45 AF548859 1 type 51 AJ467244.1 type 51 KJ467244.1 type 58 LC155228.1 type 52 KT070115.1 type 56 LC155254.1 type 66 LC155238.1 type 35 LC155246.1 type 33 AF548857.1 type 39 AB437934.1 type 5 JQ902131.1 type 68 Isolate $1-\mathrm{C}$ Isolate 2-C Isolate $3-\mathrm{C}$ Isolate 4-C Isolate 5-C

Isolate 6-C

Isolate 7-C

Isolate 8-C

Isolate 9-C

Isolate $10-\mathrm{C}$

Isolate 11-C

Isolate 12-C

Isolate 13-C

Isolate 1-U

Isolate 2-U

Isolate 3-U

Isolate 4-U

Isolate 5-U

isolate 6-U

isolate 6-U

isolate $7-U$

isolate 9-U

isolate 10-U

AF548824. 1 type 16 DQ448197.1 type 16 DQ448211.1 type 18
AF548837.1 type 18 AF548837.1 type 18 HG793863.1 type 6 KM501523.1 type 6 FN870704.1 type 11
KC470250.1 type 45 AF548859.1 type 51 KJ467244.1 type 58 LC155228. 1 type 52 КT070115.1 type 56 LC155254.1 type 66 LC155238.1 type 35 LC155246.1 type 33 AF548857.1 type 39 AB437934.1 type 59 JQ902131.1 type 68 LC155223. 1 type 16 Isolate 1-C Isolate 2-C Isolate 3-C Isolate 4-C

Isolate 5-C

Isolate 5-C

Isolate 6-C

Isolate 7-C

Isolate $8-C$

Isolate $10-C$

Isolate $10-\mathrm{C}$

Isolate 11-C

Isolate 12-C

Isolate 13-C

Isolate $1-0$

Isolate 2-U

Isolate 3-U

Isolate 4-U

Isolate 5-U

isolate $6-\mathrm{U}$

isolate 7-U

isolate $8-\mathrm{U}$

isolate 9-U

isolate $10-U$

$10 \quad 20$

30

40

50

60

70

80

90

100

TTTTGTTACTGTGGTAGATACTACACGCAGTACAAATATGTCATTATGTGCTGCCATATCTACTTCAGACCTACATATAAAAATACTAACTTTAAAGAG

…

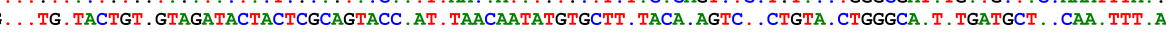
. GT . ACTGTG . TAGATAC . ACACGCAGTAC . . CATGACAT . ATGTGCA . C. GTA . CTACA . CTTCC . . A . . . CCA . TTCTG . T . . TAAAG . GTACA

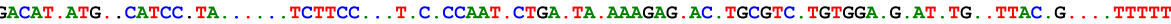
GT . ACTGTG . TAGATAC . ACACGCAGTACA. . TATGACAC . ATGTGCA. CTGTGTCTACA . CTGCT . . A . . . CTA . TTC . G. T. . TAAGG . TACA GT. ACTGTA. TGGACACTAC . CGCAGTACT. . T. TAACAT . ATGTGC. . CTACACAAA. TCCTGTGC. AAGT . . . . TG. CCCTACTAAG. TT. AGC

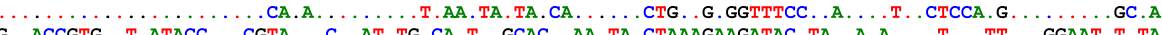

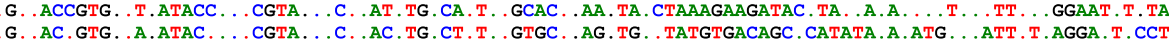
GT ACTGTA TAGATACTACT GAAGTACT C.

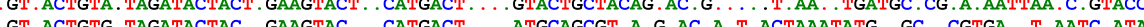
GT. AC

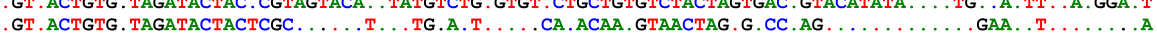
AC. . GGTA. ATACTACCCG . GTAC. ACTTT. CAT. A. TACC. . ATA. AGTCT . . TAC. TTCTA. ATATG. . СCTTY . AGTTTAAGG. T. T

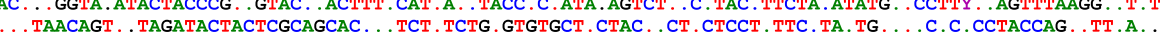
.СT. ACTGT. . TGGATAC. ACTCGCAGTAC. . . T. T. ACTT. GTCTACTACTAC. GAA. CAGCTGT. C. AA. T. . T. TG. TCCTA. TAAA. TT. AG.

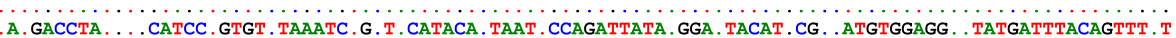

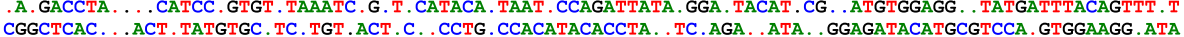

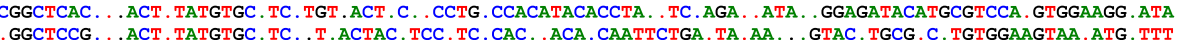
GGCTCCG . . ACT . TATGTGC. TC . T. ACTAC. TCC. TC.CAC..ACA. CAATTCTGA. TA. AA. . GTAC. TGCG . C. TGTGGAAGTAA. ATG. TTT

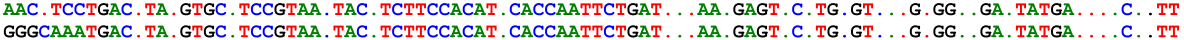
GGGCAAATGAC. TA. GTGC. TCCGTAA. TAC. TCTTCCACAT.CACCAATTCTGAT . . AAA.GAGT.C. TG.GT ...G.GG. . GA. TATGA..... . TT

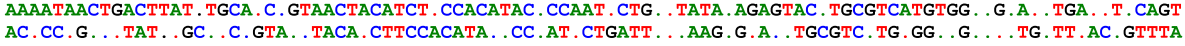
CGGCTCAC . . . ACT . TATGTGC. TC. TGT . ACT . . . CCTG. CCACATACACCTA. . TC. AGA. A ATA. . GGAGATACATGCGTCCA. GTGGAAGG. ATA

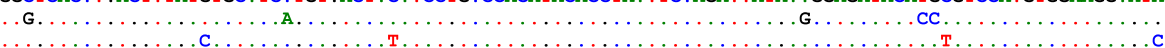

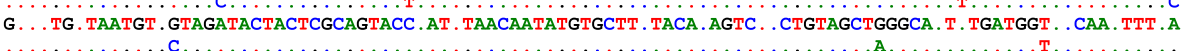

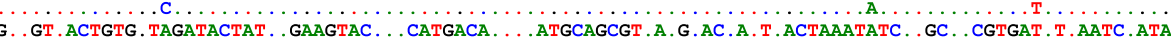
ACAAAAA . A. . ACT . . TG . G. AT . CGTA.C . . . TC. TCCA. . ACACCAA. T. TGAT. A. . AAGAGT . CATGCGTC . GTGG. AGAAT. TGA . TT . C .

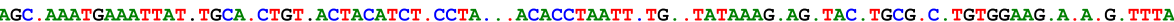
ACAATCACTGACTTAT . TGCA . C. GTAACTACATCT . CCACATAC . CCAAT . CTG . TATA . AGAGTAC . TGCGTCATGTGG . G . A. . TGA . . T. CAGT ACCACA . GACT . ATGT . CAT . CGT . ACT . CAT . TTCC . CA . ACACCAA . T . . A ATTATAAAGAG . ACATG . G . CATGTGG . G . ATA . G . T . . AC . GTTT CAC . TAGCTGACTTAT . TGCA . C. GTAACTACATCC TCCA . . ACACCAA . T . TGAT . A. . AAGAGT . CATGCGTC . GTGG . AGAAT . TGA . TT C .

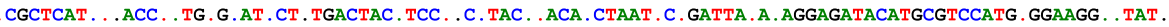

G.

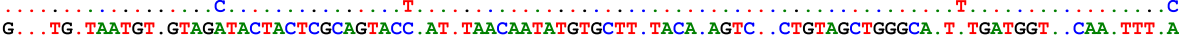
$\begin{array}{cccc}110 & 120 & 130 & 140\end{array}$ $\begin{array}{cccc}110 & 120 & 130 & 140 \\ \ldots \ldots|\ldots| \ldots|\ldots| \ldots|\ldots| \ldots|\ldots| \ldots|\ldots| \ldots|\ldots| & \ldots\end{array}$

C. GTATA.CAGACAT. TT. .G. AT . GATTTACAGT. GCAG . . TAG . GACATGTTG . GGAATA . G . TTTACAG . G G . G. GCCATGTGGA . . АGTTTGAT . T. СAGTTTA. . . . . . AGTA. GT.G.CAT.TG.AGGA. .ATGA.TT. CAG.T.A. .TA.TA.G........

CGT.ATGTTG.A.AAT.T. . C.TAC.G. . TGTT . . CA

CGTC.T.G.GA. . AATATG. . T. ACAG. TT. T . . TC

TAGACATGTGGA. . A. TATGA. . T. CAATTT . . . T

CCTTCG.C.TG. . . A.GAAT . GATT.AC.GTT.A.T.

ATTTA.G.CATG.T.A. . A. TATGAT . ACAGT . AT.

.TA....... TT.A.

AC. AGG . ACGTG. A. . AT . TG. . TTACAGTTTA

A.TA.G.C.G.CAT.TG.AGGA. .TTGA.TTGCAG.T.A.A

A. TA. . TT . GGCAT . TT . AGGA . ATGA. TTGCAA . T. A. A

TAT . . . . TTGC. TAGCATTACATTA. CTGCA.....

GAT . TAC. GT . TATTTCT . TAC... A

ACAG.T.T.TTCTCAT. A. GG

TT . TTC.A.CCCAT

.TT. TTCCA.C...

TAT . TTTCAGAA

TTT CA .

GAT . TAC. GT . TATTTCT . TAC . . . A

G. AG . TAG . GACATGATG . GCAATA G . TTTACAG

CCTTCG.C.TG...A.GAT. GAT. AC.GTT. T.

TTA. TTTTA. GAA

. GT . TATTTT. CC A.

TAT. TTTCAG

АTTT . T. AGA

. TTA. TTTTA. GAATTC

ATTTACA. TTTATTTTTCAG

G. Ag. . TAG. GACATGATg. GCAATA. G. TTtTACAg...

Fig. 3. Alignment of local isolates with reference sequences using BioEdit software. C:cervical swab, U: urine sample. 
(type 11), LC155426.1(type 33), LC155238.1 (type 35), AF548857.1 (type 39), KC470250.1 (type 45), AF548859.1 (type 51), LC155228.1 (type 52), KT070115.1 (type 56), KJ467244.1 (type58), AB437934.1 (type 59), LC15524.1 (type 66), JQ902131.1 (type 68). Figure (3) shows the alignment results using BioEdit soft. It is obvious from the figure the high diversity between both the isolates and reference sequences and among the local isolates themselves. Pairwise alignment between isolates from CSs and that from urine samples using the same software (data not shown) revealed complete coincidence of all isolates from urine samples with the correspondent isolates from CSs from the same women. Thus the further genetic analysis will be restricted to the isolates from CSs.

According the phylogenetic tree depicted in figure (4) the local isolates clustered as following: isolate number 1 clustered with accession number FN870704 type 11 (Slovenian isolate), isolate number 3 clustered with accession number HG793863 type 6 (Slovenian isolate), isolate number 4-7 clustered with accession number KM501523.1 type 6 (Thailand isolate), isolate number 9,10,12 clustered with accession number DQ448197.1 type 16 (Iranian isolate), isolate number 11 clustered with accession number AF548837.1 type 18 (Iranian isolate), isolate 13 clustered with LC1555254.1 type 66 (Kenyan isolate). On the other hand, both isolate 2 and 8 clustered with each other's and the closest reference isolate is HG793863 type 6 (Slovenian isolate).

Figure (4): Phylogenetic trees for L1 gene constructed by the neighbor joining method for 13 isolates from CSs and 19 reference isolates. Phylogenetic distances were measured by the

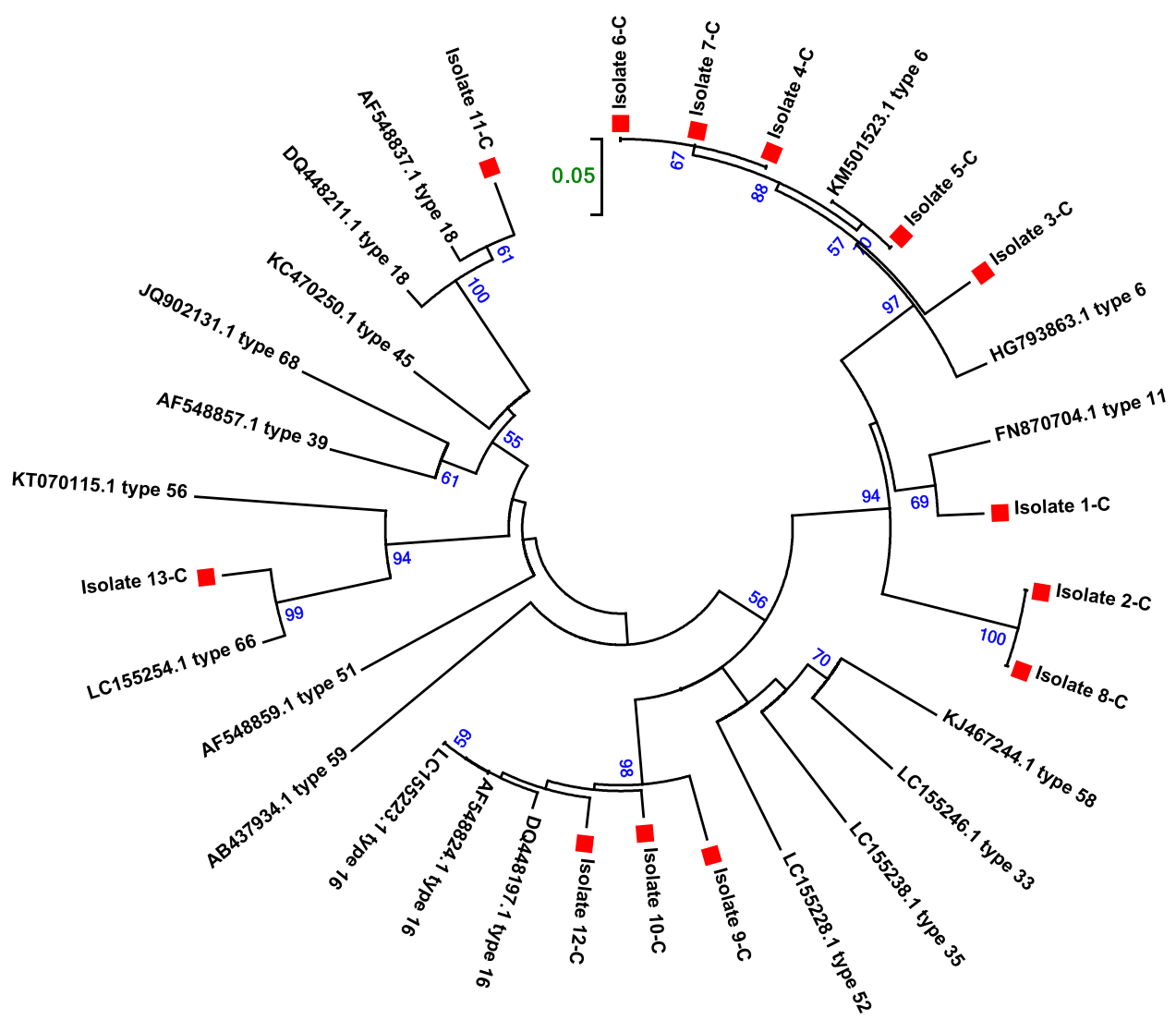

Fig. 4. Phylogenetic trees for $L 1$ gene constructed by the neighbor joining method for 13 isolates from CSs and 19 reference isolates. Phylogenetic distances were measured by the Kimura two-parameter model and the trees were statistically supported by bootstrapping with 1000 replicates. Bootstrap values below $50 \%$ are not shown. Current strains are indicated with a red square. 


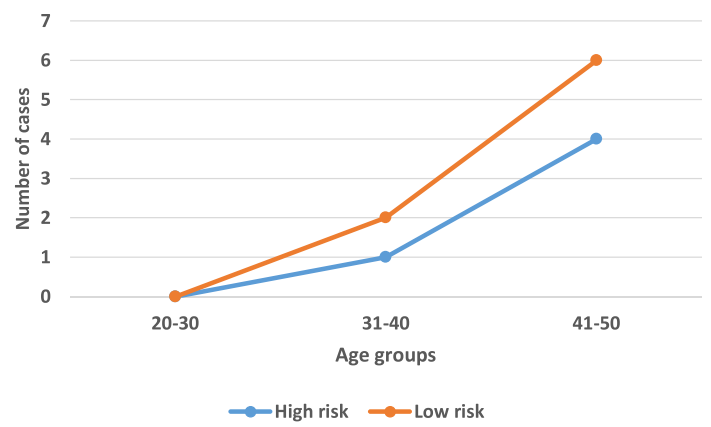

Fig. 5. Distribution of high risk and low risk HPV types in different age groups

Kimura two-parameter model and the trees were statistically supported by bootstrapping with 1000 replicates. Bootstrap values below $50 \%$ are not shown. Current strains are indicated with a red square.

\section{Association of High and Low Risk HPV Types with Different Age Groups}

According to the International Agency for Research on Cancer evaluation, out of 13 detected isolates, 8 (61.54\%) were LR HPV types, $4(30.77 \%)$ were HR HPV types and 1 isolate (7.7\%) was possible carcinogenic. For statistical purposes, the latter isolated will be considered within HR HPV. The association of high and low risk HPV types with different age groups is depicted in figure (5). Both high and low risk types increases with age and were more prevalent among age group 41-50 years.

\section{Concordance between Cervical Swab and Urine Samples in HPV DNA Detection}

Agreement in the detection of HPV DNA between CSs and urine sample was calculated via kappa statistic which was 0.853(95\% $\mathrm{Cl}=0.0 .696$ $1.01, \mathrm{P}<0.001)$, which is very good agreement. Using HPV detection in cervical samples as a reference for calculation of the accuracy of valid urine HPV results in sensitivity of $96.7 \%$ and specificity was $100 \%$.

\section{DISCUSSION}

According to the result of CSs, the current study revealed $13 \%$ as an overall prevalence of HPV infection in Iraqi women with vaginal discharge. Several previous local studies were conducted to detect the prevalence and types of HPV in Iraq. Ali et $a l .{ }^{19}$ investigated Kurdish women with different cervical lesions using a molecular method. They found $17.5 \%$ of these women were positive for different types of HPV. Fadhil et al. ${ }^{20}$ recruited 188 women with cervical dysplasia (CIN I, II, III) and used Genoarray technique for viral detection. HPV DNA was detected in $28.94 \%$ in those women, the majority of whom had HR types. What seems high proportion in these two studies compared with the current study is somewhat illusive. That is because these studies dealt with women with cervical abnormalities. Although these abnormalities are not necessarily carcinoma, they are not doubt early stage of transformation, and there is a high probability for HPV to be involved. Therefore, the reported rates may be underestimated may be due to the limited capacity of the used technique.

In contrast, the current study enrolled women with normal cervical appearance, yet relatively high rate of HPV infection was reported. This can be explained by accuracy of detection assay. The nested -PCR with two sets of universal primers can detect the vast majority of HPV, and it is considered as a gold standard for HPV detection and typing ${ }^{21}$.

There is almost a global agreement about the decisive role of screening test in the protection against invasive CC. So far, the reliable screening for HPV depend on Pap test which detects abnormal cellular change or premalignant lesions in the cervical tissue. With advent of molecular methods, there is a trend towards a direct detection of HPV from CSs. In fact, these screening methods had dramatically reduced the incidence and mortality rate of cervical cancer ${ }^{23}$.

There is almost a global agreement about the decisive role of screening test in the protection against invasive CC. So far, the reliable screening for HPV depend on Pap test which detects abnormal cellular change or premalignant lesions in the cervical tissue. With advent of molecular methods, there is a trend towards a direct detection of HPV from CSs. In fact, these screening methods had dramatically reduced the incidence and mortality rate of cervical cancer ${ }^{19}$.

Among the many drawbacks of CSs (either for Pap smear or molecular diagnosis) are the physical and psychological discomfort of women concerning pelvic examination. Moreover, the procedure is a time consuming for health care provider, and cervical cytology is susceptible for technical and subjective interpretation errors 
which may result in false negative results ${ }^{20}$. The best alternative choice for HPV is urine sample. It is a non -invasive, less expensive and more acceptable method ${ }^{21}$. If the efficiency of such alternative is proven, the screening coverage could be increased considerably primarily by reaching population in resource-limited settings ${ }^{22}$.

One of the most interesting result in current study is the very high concordance between CS and urine samples in detection of HPV DNA ( $\kappa=0.853,95 \% \mathrm{Cl}=0.696-1.01)$. To the best of our knowledge, this the first study in Iraq which used pair cervical and urine samples for HPV detection. Most result of international studies in this regard obtained encouraging findings although with some contrary. In a Spanish study including 125 women attending primary care unit for regular check -ups, Bernal et al..$^{23}$ compared the efficiency of urine samples with that of CSs in HPV DNA detection using Cobas test. The overall concordance rate was $0.76 \%, 95 \% \mathrm{Cl}=0.66-0.87$, with $90.5 \%$ sensitivity and $95 \%$ specificity. Almost similar results were reported by Tani et al. ${ }^{24}$ in 107 Italian women with abnormal cytology, and by Bissett et al. ${ }^{25}$ in 246 English women under-going routine colposcopy.

Moreover, some studies detect more genotypes in urine samples than CSs. In a recent study conducted in Thailand, Khunamornpong et al. ${ }^{22}$ used paired urine and CSs during follow -up of 168 women with previous infection with HPV. Beside the excellent concordance rate between the two samples $(\hat{e}=0.96,95 \% \mathrm{Cl}=0.9-1)$, the authors found that the absolute number of genotypes detect in urine samples was higher than that of CSs.

However, it seems that the difference between these specimens are mainly attributed to the presence of LR HPV in urine samples but not in CSs. In a Canadian study including 200 women referred to colposcopy clinic due to abnormalities in cervical cytology, vulvar, vaginal and urine samples were self-collected for HPV detection. For, HR HPV the detection rate in these samples were $86.2 \%, 62.1 \%$ and $44.8 \%$ respectively, while these rates were $53.5 \%, 62.7 \%$ and $69.7 \%$ respectively for LR HPV. The authors explained the high rate of LR HPV detected in urine samples by the susceptibility of urethral epithelial tissue but not the cervix for some LR HPV ${ }^{26}$.
In contrast some studies indicated a low detective ability of urine samples compared to CSs. In a large population-based study including 5318 women who provided with self-collected urine, cervical and vaginal samples in UK, Stanczuk et al. ${ }^{27}$ showed that urine samples had a slightly lower detection rate $(11.6 \%)$ compared to CSs (14.7\%) and vaginal swabs (16.6\%).

These variations in the result between different studies are expected. That is because a large number of factors may interfere with the results. May be the most important of these factors are urine sampling and processing.

Regarding sampling, timing, and quantity of collecting are hypothetically very important. In the current study, the first voided urine with an average of $30 \mathrm{ml}$ urine sample were used. There is a higher degree of variation between the different studies on which urine fraction is preferable for HPV DNA detection. As the aim is to analyze a maximum of exfoliate cells ${ }^{28}$, it is reasonable to use the first voiding urine as it carries the largest number of these cells compared with the other fractions.

Regarding collecting time, some studies claimed that the first urination of the day has associated with better detection of HPV DNA than other collection times ${ }^{29}$. Nevertheless, a more recent study found no significant difference in detection rate between urine samples collected at different times of day ${ }^{30}$.

Concerning urine volume, some reports stated that a large volume of urine is preferred for better detection. Burroni et al. ${ }^{31}$ used $60 \mathrm{ml}$ of urine in a study that enrolled 216 twenty-five years women in Florence/Italy. Urine samples were positive for human DNA and the concordance between these sample and CSs exceeded $85 \%$. This finding suggests a positive correlation between urine volume and exfoliated cells. However, such a large quantity of urine is not always available at one collection time.

Several other factors may affect the analytical sensitivity of HPV DNA detection in urine reviewed by Vosters et al. ${ }^{28}$. These include storage conditions, quality of DNA extraction, amplification method, and the detection of amplified DNA. During the current study, urine samples were not stored. Rather, the DNA was extracted at the same day with an average of about 2-3 hours interval 
between collection and extraction. For the other factors, the processing was performed according to the available facilities and there was a check-up after every step to ensure a fluent progress.

As a rule of thumb values of Kappa from 0.40 to 0.59 are considered moderate, 0.60 to 0.79 substantial, and 0.80 outstanding. Most statisticians prefer for Kappa values to be at least 0.6 and most often higher than 0.7 before claiming a good level of agreement. The current study reported $\kappa=0.853,95 \%(\mathrm{Cl}=0.0 .696-1.01)$, with a sensitivity of $96.7 \%$ and a specificity of $100 \%$. Almost similar result was obtained in many studies worldwide ${ }^{12,24,25}$. It implies that most HPV DNA in CSs could be detected in urine samples, while all HPV DNAs detected in urine are already have detected in CSs.

Based on the results of phylogenetic analysis for HPV DNA isolated from cervical swabs and urine samples, there were 13 isolates, 8 of which are low risk (clustered with type 6 and type 11), while other 5 isolates were HR (clustered with type 16,18 and type 66 ). Thus, $38.5 \%$ of the isolates may be considered oncogenic while $61.5 \%$ are non -oncogenic types. These current results do not agree with previous local studies ${ }^{16,17}$ in that most isolates in these studies were HR HPV.

Globally, the current results are in consistence with that obtained by papilloma Aziz et al.32 who reported high prevalence of LR HPV among Pakistani women with normal cervical cytology. The most prevalence genotypes in this study were type 6 (25\%) followed by type 55 (22.9\%) and type $11(20.8 \%)$. In the African study, on the other hand, the HR HPV genotypes were predominant with type $16,52,18$, and 51 were the most prevalence. 33 In a recent large prospective study including 1218 Iranian women with normal cervical cytology, Hamkar et al. ${ }^{34}$ found that HPV 16,18 and 66 were the most prevalent genotypes followed by type 11 and 6 .

Interestingly, most LR HPV types reported in the current study are related Slovenian or Thailand isolates, while most HR HPV types were related to Iranian isolates. For high risk types, these results are very reasonable because of geographical reasons. However, for low risk types, it appears that Slovenian isolates have a global distribution because it was frequently reported in other geographical regions like Pakistan ${ }^{32}$.

\section{CONCLUSION}

According to the results of this study, it can be conclude that The incidence rate of HPV genital infection among Iraqi child-bearing women is comparable with that of neighboring Arabic and Islamic countries. Women with late stages of childbearing age and those with a second wife are more prone for HPV infection. Urine samples can be a very good alternative for vaginal swab for HPV DNA detection from suspected women or for routine detection. The study recommends routine use of HPV detection from urine samples especially for HR HPV such as type 16 and type 18 following the steps reported in this study. As the efficiency of urine sample is proved, wide screening programs should be adopted to determine the prevalence and types of HPV using molecular methods.

\section{REFERENCES}

1. Bernard, H.U., Burk, R.D., Chen, Z., et al. Classification of papillomaviruses (PVs) based on 189 Types and proposal of taxonomic amendment. Virol. 2010;40(1):70-79.

2. Le, H.H., Bi, X., Ishizaki, A., et al. Human papillomavirus in male patients with STI related symptoms in Hanoi, Vietnam. J. Med. Virol. 2016;88(6):1059-66.

3. Denny, L., de Sanjose, S., Mutebi, M., et al. Interventions to close the divide for women with breast and cervical cancer between low-income and middle-income countries and highincome countries. Lancet 2016;389(10071):861-870.

4. Heideman, D.A., Waterboer, T., Pawlita, M., et al. Human papillomavirus-16 is the predominant type etiologically involved in penile squamous cell carcinoma. J. Clin. Oncol. 2007; 25(29):4550-4556.

5. Syrjanen, S., Lodi, G., von Bultzingslowen, I., et al. Human papillomaviruses in oral carcinoma and oral potentially malignant disorders: a systematic review. Oral Dis. 2011; 17 Suppl 1:58-72.

6. International Agency for Research on Cancer: GLOBOCAN 2012: Estimated Cancer Incidence, Mortality and Prevalence Worldwide in 2012. http://globocan.iarc.fr/Default.asp.

7. Bosch, F.X., Broker, T.R., Forman, D., et al. Comprehensive control of human papillomavirus infections and related diseases. Vaccine 2013;31 Suppl 7:H1-31.

8. Human papillomavirus and Related Diseases Report, 2017. Available at:http:// www.hpvcentre. net/statistics/reports/XWX.pdf.

9. Schiffman, M., Castle, P.E. The promise of global cervical-cancer prevention. New Engl. J. Med. 2005; 
353:2101-2104.

10. World Health Organization. Guidelines for screening and treatment of precancerous lesions for cervical cancer prevention. Geneva, Switzerland: World Health Organization; 2013. Available at: www. who.int/reproductivehealth/publications/caners/ screening and treatment of precancerous lesions/en.

11. Isidean, S.D., Coutlee, F., Franco, E.L. Cobas 4800 HPV Test, a real-time polymerase chain reaction assay for the detection of human papillomavirus in cervical specimens. Expert Rev. Mol. Diag. 2014;14: 5-16.

12. Bernal, S., Palomares, J.C., Artura, A., et al. Comparison of urine and cervical samples for detecting of human papillomavirus (HPV) with the Cobas 4800 HPV test. J. Clin. Virol. 2014;61(4):548552.

13. Vorsters, A., Micalessi, I., Bilcke, J., et al. Detection of human papilloma virus in urine. a review of the literature. Eur. J. Clinic. Microbiol. Infect. Dis. 2012;31:627-640.

14. Howard, M., Sellors, J., Kaczorowski, J., et al. Optimal cutoff of the hybrid capture II human papillomavirus test for self-collected vaginal, vulvar, and urine specimens in a colposcopy referral population. J. Low Genit. Tract Dis. 2004;8(1):3337.

15. Venceslau, E.M., Bezerra, M.M., Mota, A.C., et al. HPV detection using primers MY09/MY11 and GP5+/GP6+ in patient with cytologic and/or colposcopic changes. J. Bras. Patol. Med. Lab. 2014; 4(50):280-285.

16. Ali, K.S., Jubrael, J.M.S., Ali, H.Y.M. AOS4 detection of human papillomavirus DNA in patients with different cervical lesions in Kurdistan region, Iraq. Eur. J. Cancer 2012;48: S4-S5.

17. Fadhil, H.Y., Saleh, D.S., Al-Hamdani, F.G. Detection of human papillomavirus-21 genotypes in a sample of Iraqi women with cervical abnormalities and cancer. Canad. J. Sci. 2014;8(1);2671-2676.

18. Lee, S.H., Vigliotti, J.S., Vigliotti,V.S., Jones, W. From human papillomavirus (HPV) detection to cervical cancer prevention in clinical practice. Cancers (Basel) 2014;6(4):2072-2099.

19. Safaeian, M., Solomon, D. Cervical cancer prevention-cervical screening: science in evolution. Obstet. Gynecol. Clin. North Am. 2007;34(4):739748.

20. Jin, X.W., Sikon, A., Yen-Lieberman, B. cervical cancer screening: less testing, smarter testing. Cleve. Clin. J. Med. 2011; 78:737-747.

21. Petignat, P., Vassilakos, P. Is it time to introduce HPV self-sampling for primary cervical cancer screening. J. Natl. Cancer Inst. 2012;104(3):166-167.
22. Khunamornpong, S., Settakorn, J., Sukpan, K., et al. Comparison of human papillomavirus detection in urine and cervical samples using high-risk HPV DNA testing in North Thailand. Obstet. Gynecol. Int. 2016; 2016:6801491.

23. Bernal, S., Palomares, J.C., Artura, A., et al. Comparison of urine and cervical samples for detecting of human papillomavirus (HPV) with the Cobas 4800 HPV test. J. Clin. Virol. 2014;61(4):548552.

24. Tanzi, E., Bianchi, S., Fasolo, M.M., et al. High performance of a new PCR-based urine assay for HPV-DNA detection and genotyping. J. Med. Virol. 2013;85(1):91-98.

25. Bissett, S.L., Howell-Jones, R., Swift, C., et al. Human papillomavirus genotype detection and viral load in paired genital and urine samples from both females and males. J. Med. Virol. 2011;83(10):1744-1751.

26. Sellors JW, Lorincz AT, Mathony JB, et al. Comparison of self-collected vaginal, vulvar and urine samples with physician-collected cervical samples for human papillomavirus testing to detect highgrade squamous intraepithelial lesions. CMAJ 2000;163(5):513-518.

27. Stanczuk, G., Bater, G., Currie, H., et al. Clinical validation of hrHPV testing on vaginal and urine self-samples in primary cervical screening (cross-sectional results from the papillomavirus Dumfries and Galloway-PaVDaG study). BMJ Open 2016;6(4):e010660.

28. Vorsters A, Micalessi I, Bilcke J, Leven M, ogers J, van Damme P. Detection of human papillomavirus in urine: a review of the literature. Eur J Clin Microbiol Infect 2012;31:627-640.

29. Pathak N., Dodds J., Zamora J., Khan K. Accuracy of urinary human papillomavirus testing for presence of cervical HPV: systematic review and meta-analysis. Br Med J 2014;349 doi: 10.1136/ bmj.g5264.g5264.

30. Senkomago V, Des Marais AC, Rahangdale L, Vibat CRT, Erlander MG, Smith JS. Comparison of urine specimen collection times and testing fractions for the detection of high-risk human papillomavirus and high-grade cervical precancer. J Clin Virol 2016;74:26-31.

31. Burroni E, Bonani P, Sani C, et al. Human papillomavirus prevalence in paired urine and cervical samples in women invited for cervical cancer screening. J Med Virol 2015;87(3):508-515.

32. Aziz, H., Iqbal, H., Mahmood, H. et al. Human papillomavirus (HPV) infection in females with normal cervical cytology: genotyping and phylogenetic analysis among women in Punjab, Pakistan. Int. J. Infect. Dis. 2018;66:83-89.

33. Ogemebo, R.K., Gona, P.N., Seymour, A.J., et al. 
Prevalence of human papillomavirus genotypes among African women with normal cervical cytology and neoplasia: a systematic review and meta-analysis. PLoS ONE 2015;10(4): e0122488.

34. Hamkar, R., Shoja, Z., Ghavami, N., et al. Type- specific human papillomavirus prevalence in Iranian women with normal cervical cytology: the impact of current HPV vaccines. Intervirol 2017;60(4):125-130. 